\title{
Interaction Design for Selection and \\ Manipulation on Immersive Touch Table Display \\ Systems for 3D Geographic Visualization
}

Karljohan Lundin Palmerius and Jonas Lundberg

The self-archived postprint version of this conference paper is available at Linköping University Institutional Repository (DiVA):

http://urn.kb.se/resolve?urn=urn:nbn:se:liu:diva-160266

N.B.: When citing this work, cite the original publication.

Lundin Palmerius, K., Lundberg, J., (2019), Interaction Design for Selection and Manipulation on Immersive Touch Table Display Systems for 3D Geographic Visualization, 201926 TH IEEE CONFERENCE ON VIRTUAL REALITY AND 3D USER INTERFACES (VR), 1064-1065.

https://doi.org/10.1109/VR.2019.8798126

Original publication available at:

https://doi.org/10.1109/VR.2019.8798126

Copyright: IEEE

http://www.ieee.org/

(C)2019 IEEE. Personal use of this material is permitted. However, permission to reprint/republish this material for advertising or promotional purposes or for creating new collective works for resale or redistribution to servers or lists, or to reuse any copyrighted component of this work in other works must be obtained from the IEEE. 


\title{
Interaction Design for Selection and Manipulation on Immersive Touch Table Display Systems for 3D Geographic Visualization
}

\author{
Karljohan E. Lundin Palmerius* Jonas Lundberg ${ }^{\dagger}$ \\ Media and Information Technology \\ Linköping University, Sweden
}

\begin{abstract}
Geographic visualizations are, due to the limited need for vertical navigation, suitable for touch tables. In this poster we consider the design of interaction design for selection and manipulation through touch on the screen used for the display of 3D geographic visualization - in our case the visualization of and interaction with drone traffic over rural and urban areas-focusing on moving from a monoscopic to a more immersive, stereoscopic touch table, and how this move affects the interaction design. With a monoscopic display our stereoscopic vision uses the graphics to perceive the location of the surface, and touch interaction can naturally and intuitively be performed on top of 3D objects. Moving to stereocopic display, for increased sense of immersion, the graphics no longer provide visual cues about the location of the screen. We argue that this motivates modification of the design principles, with an alternative interaction design as a result.
\end{abstract}

\section{INTRODUCTION}

In geographic visualizations there is a limited need for vertical navigation, even when the data are presented in $3 \mathrm{D}$; a map or a $3 \mathrm{D}$ model of the ground level provides a frame of reference for the user and should therefore always be in view. Furthermore, since the navigation is mainly horizontal, over the coordinates of the map, multi-touch interaction is a popular way of providing fast and intuitive interface to such visualization applications. Such interaction even translates well into 3D, see Fig. 1.

In this poster we consider the design of interaction for selection and manipulation through touch on the screen used for the display of $3 \mathrm{D}$ geographic visualization. Our application provides visualization of and interaction with drone traffic over rural and urban areas. It has been designed and developed to test monitoring of automated air traffic and subsequent intervention of air space rules to straighten out strained situations, and does this by simulating traffic generated from pre-configured sets of services. We focus on how the design principles may change as we, in an attempt to increase the sense of of immersion, move to stereoscopic visualization, how these changes affect the interaction design and suggest a design to continue supporting natural and intuitive multi-touch interaction for selection and manipulation.

\section{Interaction on Monoscopic Touch Tables}

Natural and intuitive multi-touch interaction is achieved by mapping finger motion to object motion, as directly as possible. Many popular application, for example almost any map on a tablet, follow this prin-

*e-mail: karljohan.lundin.palmerius@liu.se

$\dagger$ e-mail:jonas.lundberg@liu.se

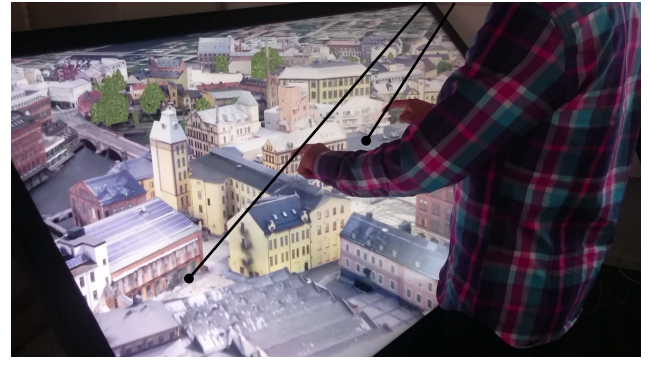

Figure 1: Touch points on a touch table with geographic visualization easily maps to points in the 3D space, as the intersection between $3 \mathrm{D}$ models or the ground plane and lines from the user's eye through the touch points.

ciple. This approach to multi-touch interaction can be formulated into these design principles:

1. touch interaction should be on the object interacted with, and

2. dragging one or two fingers over an object should transform that object so that both fingers stay on the same position on its surface.

If full 3D translation, rotation and scaling is allowed there are nine possible degrees-of-freedom to manipulate using two (one finger) or four (two fingers) degrees-of-freedom input. Nevertheless, intuitive interaction can be achieved by restricting the degrees-of-freedom being manipulated. This is done in many applications, such as $3 \mathrm{D}$ maps and 3D modelling software, by restricting the interaction, for example to in-plane translation and/or by allowing touch interaction with handles for scaling and rotation.

We have realized the aforementioned design principles into a basic design, see Fig. 2a, that we see is in line with the design of many other 3D application for touch devices. The user selects an object to manipulate by tapping on the object rendering itself with their finger or simply put their finger on it and drag the finger over the surface to manipulate its position. When selected, the object also shows handles for scaling, another detail common in 3D applications, in particular modelling software. Since the geographic visualization system has an apparent set of primary dimensions (longitude and latitude, over the map surface), we choose to primarily let finger motion affect changes within that plane. Thus, interacting with the main handles will move those handles to follow the finger at a constant height. To manipulate height there are additional, separate handles that allows movement up and down, like a slider.

\section{Interaction on Stereoscopic Touch TABLES}

With monoscopic rendering our stereoscopic vision lets us perceive the surface of the display, even when other depth cues renders the objects below or in front of the surface. With a stereoscopic display 
and rendering, on the other hand, the display can become invisible to the user. In fact, this is one of the main goals and challenges when building display systems for immersive Virtual Reality. Everyone that has by mistake run their hand into a screen trying to reach an object behind it knows that tactile contact with this invisible screen destroys the immersive illusion. For this reason we argue that touch interaction should be provided with graphics that is presented with zero parallax at the surface of the screen, that no object should be rendered in front of the surface and that the software design should discourage the user to reach for objects below the surface.

Valkov et al. have studied this problem (e.g. in [6]) and suggest moving the object to the surface before contact with the finger [5]. Other authors (e.g. in [2-4]) let the screen be a part of the user's workspace. It must then be make a natural part of the virtual environment, for example provided as a hand-held interaction panel.

However, if we do not want to force the user to keep the touch screen position in their mind during interaction but we still want tactile feedback and interaction only on top of visible objects, then we need to modify our design principles. It is no longer possible to interact by touching directly on the objects, located below the screen. Thus, another surface must be given for interaction, on the surface of the touch table. We suggest, then, these new design principles:

- each object that can be interacted with should provide an interaction surface, co-located with the touch screen, for the user to perform multi-touch interaction on,

- the interaction surface should be graphically rendered in a way that provides an intuitive link to the main object, and

- dragging one or two fingers over such a surface should transform that surface so that they stay on the same position on it, and the linked object should be transformed correspondingly.

These design principles are similar to the previous set, but postulates interaction with an intermediate interaction surface that is co-located with the touch screen instead of assuming interaction with touch contact directly on the object.

Observe that these design principles removes a dimension from direct interaction, by only providing a two-dimensional surface for interaction: in Fig. 2a a user could drag a finger on different sides of the object to move the object in different dimensions, which is no longer possible. This indicates that these principles will work best if one dimension is less important than the other two. In geographic visualization we argue that this is true, since the information is more distributed over the map than in height.

An example design that fulfil these design principles is shown in Fig. 2b, with one rectangle surface allowing 2D translation with one finger, and four handles that allow for 2D scaling of the box with a single finger. This makes the interaction similar to a puppet show, and we even considered calling it the marionette approach. Hatchet et al. suggested a similar solution [1], however in their approach they use a semi-transparent display configuration to allow rendering the 3D graphics over the touch screen, making the interaction co-located with their table.

In our design the interaction surface is a projection of the main object onto the touch screen, however not necessarily through an orthogonal projection. It would be natural in geographic visualization to make this projection in the height dimension even if the touch screen is slanted relative the ground plane, as in Fig. 1. Also, the projection may contain all kinds of visual information about more complex objects than the cuboid of our example. This can provide some of the cues necessary to let the user to associate, with low cognitive load, each interaction surface with its corresponding object, but may be combined also with lines connecting the two.

Naturally, a single finger action on the interaction surface will move the surface over the touch screen, and move the object to follow the surface on a constant height. A two finger action then

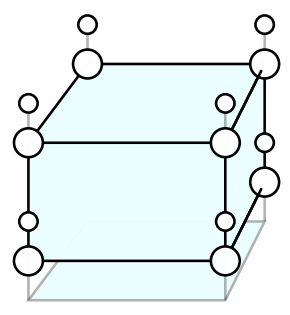

(a) Design for monoscopic display: multi-touch manipulation on surfaces and handles for scaling.

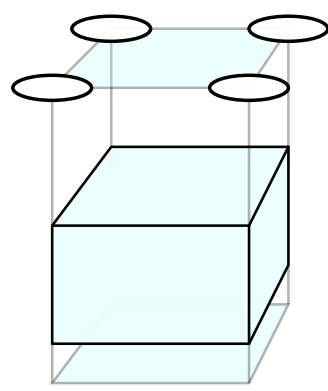

(b) Design for stereoscopic display: multi-touch interaction with a projection onto the touch surface, with handles for scaling.
Figure 2: Our suggested design for manipulation in geographic visualization without and with stereoscopy.

allows for rotation. But how about height and height scaling? In our particular application, uniform scaling is anticipated to be less common than non-uniform scaling. Because of this, the pinch-tozoom metaphor, that could be used on the interaction surface to provide uniform scaling, can instead be used to move the object in height. Think of this as pulling apart the two ends of a string that has its mid section tucked down a hole. As the ends pull apart, the mid section gets pulled out of the hole. In analogy, pulling fingers apart on the interaction surface would elevate the object towards that surface. Likewise, this string-in-a-hole metaphor can be applied on the handles to allow for vertical scaling. Thus, a two finger action on the interaction surface would move (3 DoF) and rotate (1 DoF) the object, and a two finger action on the handles would scale the object in three dimensions. It is of course also possible to provide the user with some means to switch between these two meanings of the pinch action.

\section{ACKNOWLEDGMENTS}

This study was funded by the Swedish Transport Administration and the LFV Air Navigation Services of Sweden through the project UTM CITY.

\section{REFERENCES}

[1] M. Hachet, B. Bossavit, A. Cohé, and J.-B. de la Rivière. Toucheo: multitouch and stereo combined in a seamless workspace. In Proceedings of the 24th annual ACM symposium on User interface software and technology, 2011.

[2] A. Samini and K. L. Palmerius. A perspective geometry approach to user-perspective rendering in hand-held video see-through augmented reality. In Proceedings of the 22nd ACM Conference on Virtual Reality Software and Technology, pp. 121-128. ACM, New York, NY, USA, 2014. doi: $10.1145 / 2993369.2993380$

[3] F. Steinicke, K. H. Hinrichs, J. Schöning, and A. Krüger. Multi-touching 3D data: Towards direct interaction in stereoscopic display environments coupled with mobile devices. In Proceedings of Advanced Visual Interfaces (AVI) Workshop on Designing Multi-Touch Interaction Techniques for Coupled Public, 2008.

[4] Y. Takeoka, T. Miyaki, and J. Rekimoto. Z-touch: An infrastructure for 3D gesture interaction in the proximity of tabletop surfaces. In Proceedings of ITS 2010: Devices \& Algorithms, 2010.

[5] D. Valkov, A. Giesler, and K. Hinrichs. Evaluation of depth perception for touch interaction with stereoscopic rendered objects. In Proceedings of the 2012 ACM International Conference on Interactive Tabletops and Surfaces, pp. 21-30. ACM, 2012.

[6] D. Valkov, F. Steinicke, G. Bruder, and K. Hinrichs. 2D touching of 3D stereoscopic objects. In Proceedings of ACM CHI, 2011. 